\title{
A Lyapunov Function Based Optimal Hybrid Power System Controller for Improved Transient Stability
}

\author{
R.Yousefian ${ }^{\mathrm{a}, *}$, S. Kamalasadan ${ }^{\mathrm{a}}$ \\ ${ }^{a}$ Department of Electrical and Computer Engineering, University of North Carolina at Charlotte, Charlotte, NC, USA
}

\begin{abstract}
In this paper, an intelligent power system stabilizer based on a stable and optimal hybrid learning-based adaptive control architecture is proposed which is evolved from approximate dynamic programming technique. The hybrid learning controller is based on two algorithms a) A reinforcement learning neural network controller implemented by Adaptive Critic Design (ACD) and b) A model reference adaptive controller (MRAC). The proposed method uses a novel value priority architecture for integrating these two algorithms. The designed ACD approximates nonlinear functional dynamics of the power system and interacts with the MRAC that adapts based on parametric changes. The value priority scheme developed based on a softmax network generates a hybrid control signal derived from a Lyapunov stability function that evaluates identification, performance, and stability of each controller. The overall hybrid control algorithm ensures convergence to an optimal control solution without using an explicit model of the system and at the same time ensures overall system stability. Theoretical results are validated by simulation studies for electric-generator stabilization on a 2-area 5-generator power system and as a wide-area controller on IEEE 68-bus system.
\end{abstract}

Keywords:

Adaptive Control, Adaptive Critic Design, Power System Stabilizer, Reinforcement Learning, Supervised Learning

\section{Introduction}

Modern power systems in the presence of dynamic inertia-less generators show drastic system changes and can encounter transient instability due to large excursions of rotor angles of conventional generators [1]. The interconnected dynamics of power grid along with nonlinear and time varying elements makes the stability control design of these systems complex and a challenging process. Thus, there is a specific need for developing advanced controllers that can provide system stability during normal operating changes as well as during unpredictable and nonlinear system transients. Traditionally, Power System Stabilizer (PSS) designed using conventional linear leadlag structure [2], adaptive control [3], feedback linearization techniques $[4,5]$, etc. provides damping of oscillation modes. However, it is a well known fact that the response of a dynamic nonlinear system cannot be tracked using a linear model-based controller. For instance, adaptive control design is capable of adapting to the normal varying operating points only when the variations can be represented as a linear parametric set [3] as these controllers are not designed for nonlinear uncertainties especially in the Wide-Area Control (WAC) designs.

\footnotetext{
*Corresponding author. Tel.: +1 7043520154

Email addresses: ryousefi@uncc.edu (R.Yousefian), skamalas@uncc.edu (S. Kamalasadan)

${ }^{1}$ This work was supported in part by National Science Foundation
} under the grant NSF Grant ECS-1229598 and ECS- 1309911.
It has been proven that, learning-based controllers can effectively learn and map the system dynamics from input/output space relationship without any prior knowledge of the system, thus effectively dealing with the limitations of model approximation [6]. In the online process, these controllers revive a learned policy in the presence of an associated input or generalizes for a new input. Neural Network (NN) as a distinct learning-based function approximator has been effectively implemented as a power system intelligent controller in several works [7-9], and their ability to adapt during nonlinear transient conditions have been discussed [7-12]. These architectures use NNs in the form of supervised learning as an intelligent PSS for damping generator oscillations. However, majority of these works have used the intelligent controller by itself. For such designs perfect training of NNs are required for overall stability which may not be achievable all the time.

Augmentation forms (learning based schemes augmenting linear controllers), without consideration of the impacts of these controllers on each other are also studied before. Our earlier work [12] illustrated the concept of system supervision where NN controller augments a conventional MRAC. In that design, parametric and functional adaptation has been performed, and a controller that can be integrated with conventional PSS was developed and illustrated. Also, different controller configurations are used as a hybrid design based on changing system dynam- 
ics, such as gain scheduling and switching technique. For example, combination of a linear and NN-based nonlinear adaptive controller through switching law is studied in [13]. An advanced reconfigurable controller enhanced by multiple model architecture is proposed in [14]. Even though supervised learning-based NNs achieve impressive control performance after learning, they are not guaranteed to achieve global optimal solution until the learning process is complete.

Another distinct method in this area is Reinforcement Learning (RL), which can improve tracking performance through repeated trials of interaction with the system thus overcoming the need for complex nonlinear designs [15]. Adaptive Critic Designs (ACDs), originally proposed in [16], is one of the techniques designed for RL by performing Approximate Dynamic Programming (ADP) forwardin-time. In general, this architecture performs optimal control theory through adaptation of nonlinear function approximators, mainly NNs. The main advantages of the RL over supervised learning technique is its ability to: be used in learning problems involving sequential dynamics, optimize a scalar performance objective, and reduce the computational burden of offline training process. However, the heuristic nature of this type of controllers still causes stability issues; thus cannot be fully trusted in real-life implementation even tough being tested in real-time simulations $[7,8]$. Therefore, the need to develop a more advanced hybrid design that provides stable control actions under unforeseen disturbances is very clear and that is the main focus of this paper.

In this paper, we propose a novel learning-based scheme for power system control that uses ADP methodology. The approach built upon [17] and inspired by [18, 19] is based on the combination of experts to enhance optimality of the control and generality of NN learning. Our choice of performance criterion is on the basis of direct Lyapunov stability analysis that encourages minimization of the composite identification and closed-loop errors. The uniqueness of the proposed method is its softmax value priority network that makes sure that the closest stable optimal solution at a given time horizon is achieved. The linear MRAC works well especially when the control objective is to make the output follow a particular path, and the NN controller is employed in tracking the unknown nonlinearity and uncertainity of the system. The learning-based controller seamlessly evolves based on system changes from a simple supervised structure to a full RL-based controller. Moreover, additional source of exploration is provided that eliminates the necessity of offline training for RL. The proposed method allows the system to dynamically shift between linear and intelligent controllers and thus can be effectively utilized on any practical set up. The global controller can also interact between two or more local controllers thus operating as a wide-area control system.

The remainder of the paper is organized as follows. Next section provides a brief overview of the proposed method. In section 3, mathematical preliminaries and foundation of the proposed method is provided. Section 4 presents the control methodology using value priority. Implementation and simulation results on the test power system is presented in section 5. Section 6 presents the implementation results of the proposed architecture as a WAC, and section 7 concludes the paper.

\section{Learning-based Adaptive Controller Topology}

Fig. 1 shows the block diagram for the proposed hybrid learning-based adaptive control topology. As demonstrated in the figure, the control architecture develops a signal $u$ (a combination of an adaptive controller signal $u^{a d}$ and a neuro-controller $u^{r}$ ) that can control the system in presence of parametric changes and functional nonlinearities and uncertainties. Both controllers are updated based on a specific adjustment mechanism developed using a reference model. For the adaptive controller, reference model generates a stable reference output to be tracked by the system. The adaptation rule is on the basis of a Lyapunov function, a linear model of the system, and the closedloop error. As a measurement-based tool, neuro-controller is updated based on RL algorithm and ACD technique. In this method, using the Identifier NN, the Critic NN for current and future time steps, and controller NN, the optimal cost function in approximated. The combined identified and reference model consist of utility and cost functions that are optimized in a predictive manner. The adaptive controller, here MRAC, forces the system output to track the reference model during parametric changes. Theoretically this task bounds the deviation in the system function. Thus the NN RL learns the system functional changes in this bounded region. The main advantage of this approach is that NNs can perform the functional adaptation without excessive tuning and saturation.

The value priority block acts as a manger to generate a performance trajectory, which tracks the stability and optimality of the closed-loop system. Receiving the data needed, RL performance is first compared to adaptive controller with respect to optimal action backward-in-time. Further, identification and stability indexes are calculated in Lyupunov function and input-output stability framework. The approach thus guarantees quadratic stability of the system $\left(\dot{J}_{k}^{a d}<0\right)$, while improving optimal performance $\left(\frac{\partial J_{k}^{r}}{\partial u_{k}^{r}}=0\right)$. Overall, the technical issues addressed by the hybrid architecture are: allocating different networks to learn different tasks based on value priority probabilistic rules, detecting the RL training patterns and operating regions, and shifting the control output toward RL as it has gained knowledge from the system and the linear controller.

\section{Mathematical preliminaries}

\subsection{System Identification, Energy Function, and Control}

Let $x \in \mathbb{R}^{n}$ and $u \in \mathbb{R}^{m}$ denote the system states and control inputs vectors, respectively. The discrete system 
Adjustment Mechanism

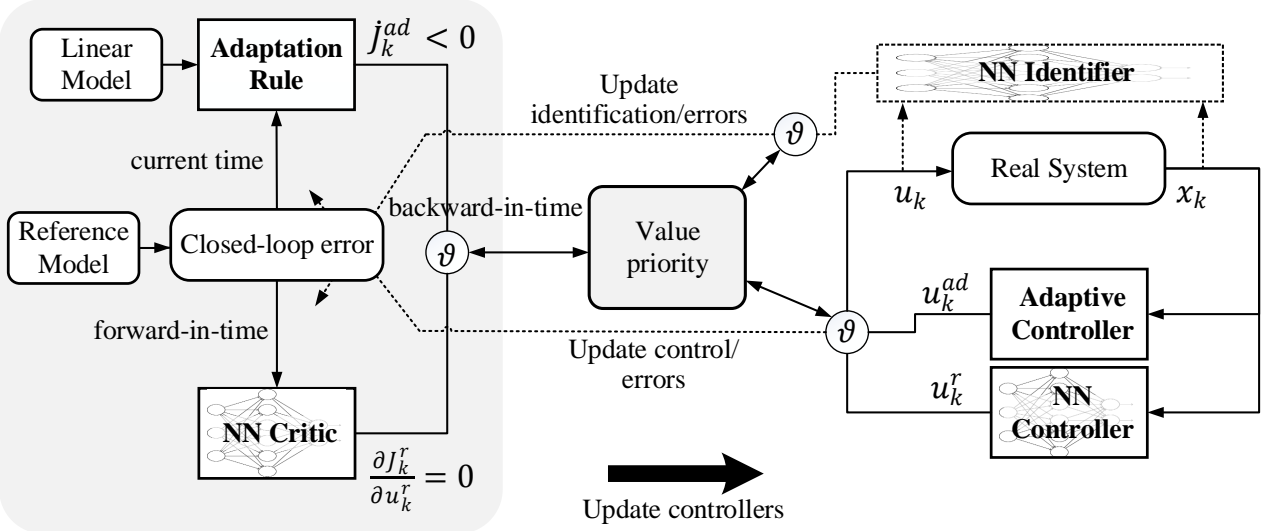

Figure 1: Learning-based Adaptive Controller Topology

dynamics are separated into two components of a linear parametric and an unknown nonlinear functional adaptation. Consider a general class of nonlinear system as

$$
x_{k+1}=\underbrace{A x_{k}+B u_{k}}_{\text {linear model }}+\underbrace{f\left(x_{k}\right)+g\left(x_{k}\right) u_{k}}_{\text {nonlinear model }}+d_{k}
$$

where, $k$ refers to the $k$ th time step, $f(.) \in \mathbb{R}^{n}$ and $g(.) \in \mathbb{R}^{n \times m}$ are unknown bounded nonlinear functions and $f(0)=0$ and $g(0)=0, A \in \mathbb{R}^{n \times n}$ and $B \in \mathbb{R}^{n \times m}$ are the unknown parametric change, and $d$ is unknown and bounded uncertain disturbance.

The goal of this paper is to find an estimate of $A, B, f($.$) ,$ and $g($.$) and an associated confidence interval of this esti-$ mation from measurement data. This information is furthermore used to design a hybrid adaptive nonlinear controller that stabilizes the system despite the uncertainty in the estimates. In general, the control task is to determine optimal control law for the system state vector $x$ to converge the system from a known initial state $x_{0}$ to a new stable equilibrium $x^{*}$. To accomplish this task, we use a linear and nonlinear direct energy function methods, which focuses on the transient dynamics of the system. In other words, the goal of the paper is to develop computationally tractable optimal control and transient stability cost function policy guaranteeing system convergence to the optimal equilibrium. The energy function of the overall system (1) can be defined as a superposition of linear and nonlinear Lyapunov functions proposed on tracking error $\tilde{x}=x-x^{*}$ as,

$$
J\left(x_{k}\right)=\tilde{x}_{k}^{T} P \tilde{x}_{k}+J^{n l}\left(\tilde{x}_{k}\right)
$$

The first element in the above equation refers to energy of the linear part. The second element, $J^{n l}$, is a function mapping $\mathbb{R}^{n}$ to $\mathbb{R}$ for nonlinear Lyapunov function $[20,21]$. It can be proved that the system is asymptotically stable in the sense of Lyapunov stability criteria such that:

$$
J\left(x_{k}\right) \geq 0, \quad \Delta J\left(x_{k}\right) \equiv J\left(x_{k}\right)-J\left(x_{k-1}\right) \leq 0
$$

For this, the weighting matrix $P$ is of appropriate dimension and required to be positive-definite $\left(\lambda_{i}(P)>0\right)$, and,

$$
A P+P A^{T}+Q_{0}=0
$$

where $Q_{0}>0$. Also, $f(x)$ in (1) should satisfy $f(x)^{T} N x>$ 0 for some constant real matrix $N, J^{n l}(x) \geq 0$, and $\nabla J^{n l}(x)=Q^{T} f(x)$ for some constant real matrix $Q$ [20]. Then,

$$
\Delta J\left(x_{k}\right)=\tilde{x}_{k}^{T} Q_{0} \tilde{x}_{k}+u_{k}^{T} R u_{k}+\tilde{x}_{k}^{T} \operatorname{Pf}\left(x_{k}\right)+Q^{T} f\left(x_{k}\right) \Delta x_{k}
$$

where $\Delta x_{k}=x_{k}-x_{k-1}$. Overall, as long as the effect of nonlinearity is relatively small and bounded, smaller cone of Lyapunov function can be defined to clarify the stability of $x^{*}$ and estimate the domain of attraction [21].

To this end it is desired to find the control policy $\pi=\left\{u_{0}, u_{1}, \ldots\right\}$ that satisfies the aforementioned Lyapunov stability criteria. Moreover, an optimal control policy $u^{*}$ can be found that minimizes the infinite-horizon cost function $J_{\pi}\left(x_{0}\right)$. Considering that dynamics of the system can be identified, controller action can be presented as linear and non-linear parts. Denoting $u^{a d}$ as initial controller around the plant equilibrium point which is adaptive in nature, a nonlinear-based controller can be used to approximate the system functional dynamics. The RL-based controller can be activated to learn the nonlinear subsystem dynamics of $f($.$) and g($.$) for which no prior knowledge$ is available.

\subsection{MRAC Adaptation}

In general, MRAC works so that the state vector $x$ tracks a desired state vector as specified by the reference input under the condition of the state vector remaining bounded. Let the tracking reference model be represented as

$$
x_{k+1}^{r e f}=-A_{m} x_{k}^{r e f}+B_{m} r_{k}
$$

where, $r$ is the input command, and $A_{m}$ and $B_{m}$ are reference coefficient matrices. In this paper, direct adaptive approach is employed, where plant parameters are considered as known and the control parameters are adjusted directly to minimize the closed-loop error between system and reference model states [22]. Based on (1), the firstorder linear time-invariant system can be described by

$$
x_{k+1}^{a d}=A x_{k}+B u_{k}^{a d}
$$




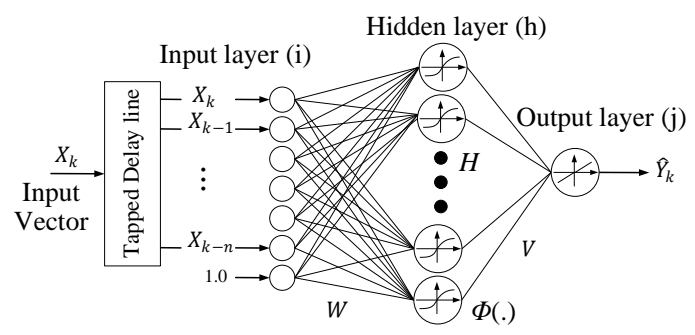

Figure 2: Feed forward neural network structure

With the knowledge of system dynamics, an adaptive linearizable control feedback can be estimated as

$$
u_{k}^{a d}=\theta_{x} x_{k}+\theta_{r} r
$$

The closed-loop error equations for $\tilde{x}=x-x^{r e f}$ can then be derived in terms of adaptation parametric set of $\theta=$ $\left[\theta_{x}, \theta_{r}\right]^{T}$ as,

$$
\tilde{x}_{k+1}=-A_{m} \tilde{x}_{k}+B \tilde{\theta}_{x} x_{k}+B \tilde{\theta}_{r} r
$$

where, $\tilde{\theta}=\theta-\theta^{*}$ are controller parameter error. An adaptive law can be generated based on Lyapunov function candidate to converge these estimates to the actual parametric values denoted with the superscript $*$. At every time step, the aim is to determine adaptive law for $\theta$ updating continuously so that the overall system has bounded solution and the state error $\tilde{x}$ tends to zero asymptotically. This adaptive law can be derived as,

$$
\Delta \theta_{x}=-\operatorname{sgn}(B) \tilde{x} x, \quad \Delta \theta_{r}=-\operatorname{sgn}(B) \tilde{x} r
$$

where $\Delta \theta_{k}=\theta_{k}-\theta_{k-1}$ is the parameter update at time step $k$. From the definition of the closed-loop error and adaptive laws it can be shown that a Lyapunov function exists as linear parts of (2). This proves the stability of the controller. It should be noted that an additional augmentation of growth rate of parameters can also be used to ensure the boundedness of the signals [22].

\subsection{ACD Adaptation as Reinforcement Learning}

In general, ACDs can perform optimal control policy for a given system based on RL by means of ADP principles. Essence of ACD is approximation of the HamiltonJacobi-Bellman (HJB) equation associated with optimal control theory and meeting a certain objective function, here, Lyapunov stability function [6]. The HJB equation is developed using $\tilde{x}=x-x^{r e f}$ around the reference point for present time step and future window. System nonlinear identification is carried out by means of adapting Feed Forward Neural Network (FFNN) presented in Fig. 2, where the output values are computed by inner product between the estimated weight vector $\hat{W}$ and a state-dependent feature vector $\Phi($.$) . It can be represented as$

$$
\hat{x}_{k+1}^{r}=\hat{W}_{I}^{T} \Phi_{I}\left(V_{I}^{T}\left[x_{k}, u_{k}\right]\right)+\epsilon_{I}\left(x_{k}, u_{k}\right)
$$

where, $\Phi \in \Re^{h}$ is the corresponding nonlinear mapping function of the states which is usually considered as sigmoidal function, $W \in \Re^{h, j}$ and $V \in \Re^{i, h}$ are the parameter vector of weights of the FFNN where $i, h, j \in \mathbb{N}$ are dimensional space of the feature vector representing the input, hidden, and output layers of the NN, and $\epsilon$ is the $\mathrm{NN}$ functional approximation error. If we fix the first-layer weights, then NN has only one layer of tunable weights. Thus the identification error can be defined as,

$$
e_{k}^{I}=x_{k}-\hat{W}_{I}^{T} \Phi_{I}\left(V_{I}^{T}\left[x_{k}, u_{k}\right]\right)
$$

Our controller is based on the assumption that the NN structure can be used to approximate any nonlinear function. To this the following Lemma holds.

Lemma 1. For any $N N$ structure, it is guaranteed that due to the universal approximation property there exists a set of bounded weights $W$ and output functions $\phi$ such that the nonlinear inversion error $\epsilon$ can be approximated over a compact set $D \subset \Re \times \Re^{n}$ by a weighted parameter set.

ProOF. [23, p. 52]

The design of an optimal controller is based on the fact that the cost function or policy approximation is converged to a best optimal value, $J^{*}$, with an optimal control action, $u^{*}$. In the ACD architecture this task is performed by successively adapting two NNs so-called critic NN and action $\mathrm{NN}$, which respectively, learn the desired cost function and desired control value based on the estimated cost function. In this paper, HDP has been used as an ACD technique, in which, critic network represents the cost function by being trained explicitly and directly on the states of the system. These models approximate the control action $u^{r}$ and the cost function $J^{r}$, respectively as,

$$
\begin{aligned}
& \hat{u}_{k}^{r}=\hat{W}_{A}^{T} \Phi_{A}\left(V_{A}^{T} x_{k}\right)+\epsilon_{A}\left(x_{k}\right) \\
& \hat{J}^{r}\left(x_{k}\right)=\hat{W}_{C}^{T} \Phi_{C}\left(V_{C}^{T} x_{k}\right)+\epsilon_{C}\left(x_{k}\right)
\end{aligned}
$$

where $J^{r}$ represents the nonlinear mapping in (1). It is required to $J^{r}\left(x_{0}\right)=0$ as a boundary condition to serve as a Lyapunov function [24].

\section{Hybrid Control Structure}

In this section we seek an answer to the question of existence of a value priority network that learns the operating region of controllers as a function of performance and identification. This will allow us to build a hybrid controller that provides quadratic stability, while improving the performance of the overall closed loop system. The architecture utilized in this paper to perform the value priority shifting policy is proposed based on a supervised RL structure and mixture of experts presented in Fig. 3. The $\mathrm{RL}$, the expert and the value priority networks form a hybrid scheme that provides a composite control action to the system as,

$$
u_{k}=\vartheta_{k}^{a d} u_{k}^{a d}+\vartheta_{k}^{r} u_{k}^{r}
$$

where, $\vartheta^{r}$ and $\vartheta^{a d} \in[0,1]$ are value priority trajectory of controllers. The RL network competes with the expert to learn the training patterns and the value priority network 


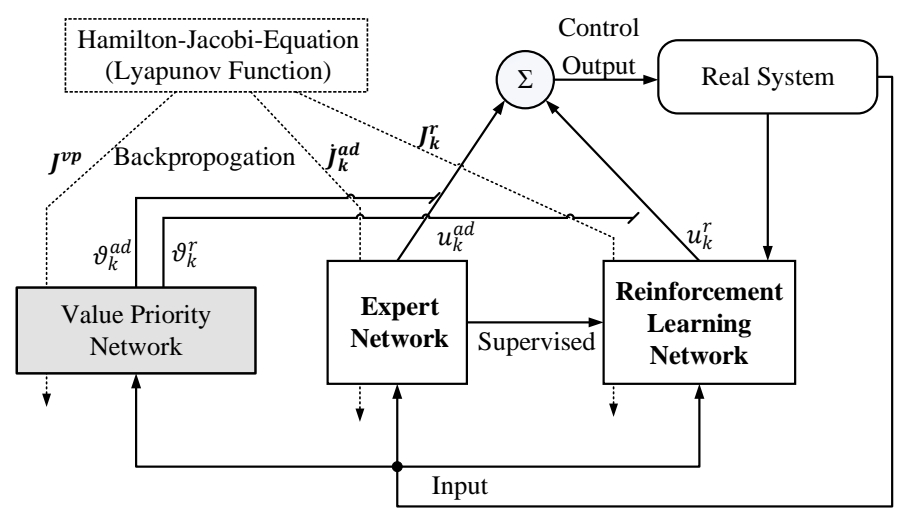

Figure 3: Hybrid control structure with value priority network

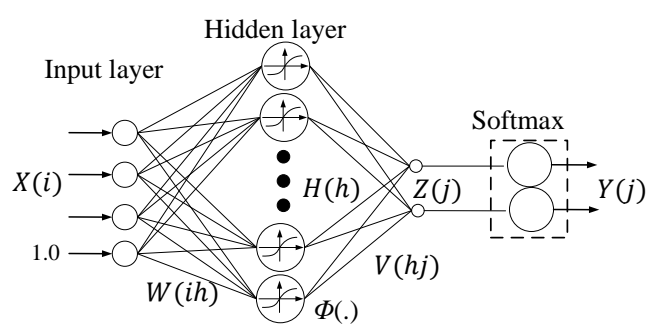

Figure 4: Value priority network structure

mediates this competition. The learning policies used to train the RL and the value priority networks are based on the minimization of HJB equation, respectively, forward and backward in time. For this, intuition is expressed mathematically as follows.

\subsection{Value Priority Network Update}

As discussed before, the value priority computes the action, $u$, as a weighted sum of the actions received by both component policies, $u^{r}$ and $u^{a d}$. The activation of these output units must be non-negative and sum to one. To meet these constraints, we use Softmax activation function [19] at the output layer of the FFNN as depicted in Fig. 4 and represented as,

$$
\vartheta^{r}=e^{z^{r}} /\left(e^{z^{r}}+e^{z^{a d}}\right), \quad \vartheta^{a d}=1-\vartheta^{r}
$$

where $z$ is the total weighted inputs received by the corresponding output as,

$$
z=\hat{W}_{v p}^{T} \Phi_{v p}\left(V_{v p}^{T} x\right)
$$

The training value priority error function for the controllers, $J^{v p}$ is derived from the Lyapunov stability function of the system, (1). Evaluating the total energy, which is the cumulative effect of changes in the energy $\Delta J$ from previous states and the two control actions, the sensitivity of each controller with respect to the total energy can be extracted. Hence, the priority of each controller that reduces this energy function can be learned. The proposed function is combination of the identification and controllers performance as,

$$
J_{k}^{v p}=\sum_{n=0}^{k} \lambda^{k-n}\left(e_{n}^{I} Q_{0} e_{n}^{I}+\tilde{u}_{n} R \tilde{u}_{n}\right)
$$

The first part is a measure of the identification error of the controllers over a finite backward window $n \in(0, k]$ in order to distinguish the operating region. The significance of this element as a part of value priority function is that, as the error of identification for RL gets smaller, it can provide closer to optimal estimate of the cost and control policies. On the other hand, if the system is known to MRAC, it can take control of the system.

The second part is the performance function developed on $\tilde{u}_{n}=u_{n}-u_{n}^{*}$ as the estimated control error at time step $n$. In order to derive this element, quality of the controllers is iteratively evaluated with respect to $u^{*}$. Towards this, considering the current time as the terminal state, calculations are done backward for $J$ and $u$ as,

$$
\begin{aligned}
J_{i}\left(x_{n}\right) & =U\left(x_{n}, u_{n i}\right)+J_{i}\left(x_{n+1}\right) \\
u_{n i} & =\underset{u}{\operatorname{argmin}}\left(J_{i}\left(x_{n}\right)\right)
\end{aligned}
$$

where, $i$ is iteration number indicating the number of times the window has been swiped, and $u_{n}$ is estimated as,

$$
\begin{aligned}
u_{n} & \approx R^{-1}\left(\frac{\partial x_{n+1}}{\partial u_{n}}\right)^{T} \frac{\partial U\left(x_{n+1}, u_{n+1}\right)}{\partial x_{n+1}} \\
& \approx R^{-1}\left(\frac{\partial x_{n+1}}{\partial u_{n}}\right)^{T}\left(Q_{0} x_{n+1}+R u_{n+1} \frac{\partial x_{n+1}}{\partial u_{n}}\right)
\end{aligned}
$$

The state estimation is carried on in forward manner, to estimate the new trajectory and sensitivity by

$$
\begin{aligned}
& x_{n+1}=\vartheta_{k-1}^{a d}\left(A x_{n}+B u_{n i}\right)+\vartheta_{k-1}^{r}\left(f\left(x_{n}\right)+g\left(x_{n}\right) u_{n i}\right) \\
& \frac{\partial x_{n+1}}{\partial u_{n}}=\vartheta_{k-1}^{a d} B+\vartheta_{k-1}^{r} g\left(x_{n}\right)
\end{aligned}
$$

It is assumed that the estimation of the model is accurate enough for the visited states based on identification value priority criteria, and the HJB equation converges to a solution in this window. If we differentiate the value priority function with respect to the outputs of the value priority network we get a signal for training the network. Overall, the problem formulation requires that whenever there is a system trajectory movement towards RL, then $\vartheta^{r}$ is raised, meaning that the RL has learned the optimal cost function and generates less than the average squared error of the controllers. The rate of this learning is

$$
\frac{\partial J^{v p}}{\partial z^{r}}=\vartheta^{r}\left(J^{v p \mid r}-J^{v p \mid a d}\right)
$$

Furthermore, back-propagation of this error function yields update to the weights, which generate the value priority trajectory. The hybrid shifting rule is initialized by $\vartheta_{0}^{r}=0$ as representation of adaptive control activating the system.

\subsection{Operating Region and Identification Update}

The goal of the hybrid control problem considered in this paper is to stabilize the system around a fixed desired operating point through the energy function minimization. Due to the fact that a priori operating points are given 
by the adaptive controller and associated steady-state inputs are known, we can iteratively update the operating point for the unknown dynamics of the ACD controller as $\tilde{x}_{k}^{r}=x_{k}-x_{k}^{r e f}$. Hence, for the ACD combination, the reference model output will act as a predictive stable reference output. The neuro-identifier predicts plant output that needs to be tracked at time $k+1$, and the action $\mathrm{NN}$ will generate control signal accordingly in a predictive manner.

Furthermore, the goal of RL is to eventually evolve to learn both linear and nonlinear regions. This yields to operating region of $\hat{x}_{k+1}=\vartheta^{a d} \hat{x}_{k+1}^{a d}+\vartheta^{r} \hat{x}_{k+1}^{r}$. The aforementioned rule thus makes the action $\mathrm{NN}$ independent of the adaptive controller in the case of learned scenarios.

\subsection{Supervised Reinforcement Learning Update}

In this part we present an iterative supervised ADP algorithm, where the cost function and the control law are updated recursively. In the proposed method the costto-go function is given based on the Lyapunov stability function as follows:

$$
J^{r}\left(x_{k}\right)=\sum_{n=k}^{\infty} \gamma^{n-k} U\left(x_{n}, u_{n}\right)
$$

where, $U$ is the utility function and $\gamma$ is the discount factor [8]. An explicit utility function is used for reward/punishment for RL, or as incremental cost function in Lyapunov stability concept based on,

$$
U\left(x_{t}, u_{t}\right)=\tilde{x}_{t}^{T} Q_{0} \tilde{x}_{t}+u_{t}^{T} R u_{t}+Q^{T} f\left(x_{k}\right) \Delta x_{k}
$$

which is equal to $\Delta J_{k}$ in (5) with neglection of linear and nonlinear interaction element.

The process of training a NN, as the implementation benchmark of this method, requires computing an error value that describes how the NNs output varies from the target value. Time adapted back-propagation algorithm is widely used in training NNs for this purpose. This method thus allows us to calculate the sensitivity of each component of the NN to the error and minimize it [6]. In particular, the training process of the critic NN is based on ADP forward-in-time, which estimates $J^{*}$ by updating its policy with respect to critic error as,

$$
e^{C}=\hat{J}^{r}\left(x_{k}\right)-\gamma \hat{J}^{r}\left(\hat{x}_{t+1}\right)-U\left(x_{k}, u_{k}\right)
$$

with elements of the rewards obtained from the system, the cost functions at current time step and future time step, estimated by (14). The critic NN future outputs is based on predicted states derived from $\mathrm{NN}$ and adaptive identification as discussed before. The action NN training is based on minimizing the derivative of this estimated cost function to control action chosen. The purpose is to bring the action NN error asymptotically to zero in an iterative process. This can be derived as,

$$
e^{A}=\frac{\partial U\left(x_{k}, \hat{u}_{k}\right)}{\partial u_{k}}+\frac{\partial \hat{J}^{r}\left(\hat{x}_{k+1}\right)}{\partial \hat{x}_{k+1}} \cdot \frac{\partial \hat{x}_{k+1}}{\partial \hat{u}_{k}}
$$

In the proposed method, the value priority parameter, $\vartheta$, not only provides $u$, but also plays an important role to modify the RL's policy as well. After each state transition, the parameters of RL are updated according to the update rule derived as

$$
\begin{aligned}
& \Delta W_{C}=\alpha\left[\vartheta^{r} e^{C}+\vartheta^{a d}\left(J^{a d}-J^{r}\right)\right] \nabla_{W_{C}} J^{r} \\
& \Delta W_{A}=\alpha\left[\vartheta^{r} e^{A}+\vartheta^{a d}\left(u^{a d}-u^{r}\right)\right] \nabla_{W_{A}} u^{r}
\end{aligned}
$$

where, $\alpha$ is small step size learning parameter, $J^{a d}$ and $u^{a d}$ are adaptive references for Critic and Action NNs. This feature leads the RL controller to merge faster towards supervised controller especially early in the learning process when the critic has a poor estimate of the optimal cost function based on the value priority criteria. Furthermore, it is proven that the update method for the proposed structure of supervised RL yields to optimal value and is converging to a compact optimal set.

Theorem 1. Let $J_{i}^{r}$ and $u_{i}^{r}$ be the RL-based controller input sequence at each iteration $i$, which performs weights updates per (28) and (29). Assuming,

$$
J_{0}^{r}\left(x_{k}\right)=0 \leq J^{a d}\left(x_{k}\right) \leq J^{*}\left(x_{k}\right) \leq J^{\max }\left(x_{k}\right)
$$

It can be proved that as $i \rightarrow \infty, J_{i}^{r}\left(x_{k}\right) \rightarrow J^{*}\left(x_{k}\right)$ and $u_{k i}^{r} \rightarrow u_{k}^{*}$. Hence, the sequence of $J_{i}^{r}$ converges to the solution of the HJB and the hybrid controller is stable.

Proof. See Appendix.

\section{Implementation as a Power System Stabilizer}

Let the rotor angle and speed be the states of generator $i$ represented as $x=\left[\delta_{i}, \dot{\delta}_{i}\right]$. In order to address the Lyapunov stability criteria and transient energy, we use a traditional swing equation dynamic model of a synchronous generator expressed as [21],

$$
M_{i} \ddot{\tilde{\delta}}_{i}+D_{i} \dot{\tilde{\delta}}_{i}+\sum_{j} K_{i j} \sin \left(\delta_{i j}\right)-P_{m i}=0
$$

where, $\tilde{\delta}_{i}=\delta_{i}-\delta_{i}^{*}, \delta_{i j}=\delta_{i}-\delta_{j}, M$ is the inertia constant of the synchronous generator, $P_{m}$ the reference or mechanical power, $D$ damping coefficient, and $K_{i j}=B_{i j} V_{i} V_{j}$ with $B_{i j}$ being the susceptance matrix between buses of $i$ and $j$, and $V_{i}$ is the terminal voltage at bus $i$. It can be further shown that $P_{m}$ satisfies the the post-fault equilibrium point as $P_{m i}=\sum_{j} K_{i j}^{*} \sin \delta_{i j}^{*}$. Hence, the nonlinear dynamics can be expressed as,

$$
f\left(\delta_{i}\right)=\sum_{j} K_{i j} \sin \left(\delta_{i j}\right)-K_{i j}^{*} \sin \left(\delta_{i j}^{*}\right)
$$

If the changes of the generator states are in small-signal domain it could be linearized around the operating point leading to $f\left(\delta_{i}\right)=\sum_{j} K_{i j} \delta_{i j}-K_{i j}^{*} \delta_{i j}^{*}$, well-suited for MRAC control; whereas, in the case of severe transient changes, where the post-fault points are fallen into nonlinear region, NNs are triggered to gain the control of the system. Nonlinear energy function can be described as 


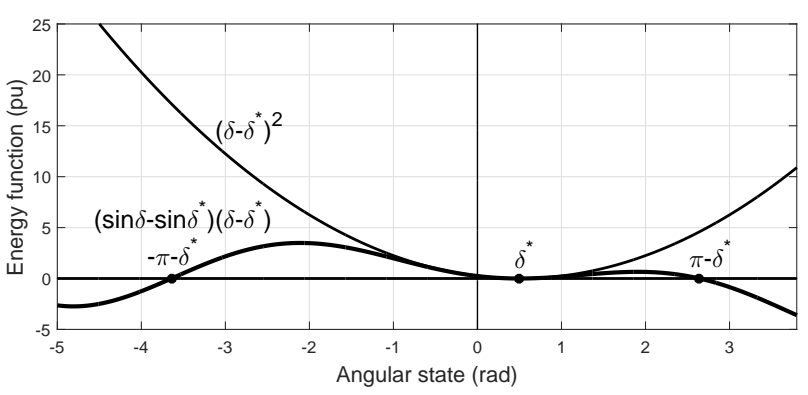

Figure 5: Boundedness of nonlinear energy function.

a function of angular differences with the assumption of $K_{i j}^{*}=K_{i j}$ as,

$$
\begin{aligned}
J^{n l}\left(\delta_{i}\right) & =\int_{0}^{\tilde{\delta}_{i}} f(\delta) d \delta \\
& =-\sum_{j} K_{i j}\left[\cos \left(\delta_{i j}\right)-\cos \left(\delta_{i j}^{*}\right)-\left(\delta_{i j}-\delta_{i j}^{*}\right) \sin \left(\delta_{i j}^{*}\right)\right]
\end{aligned}
$$

This definition yields to $\Delta J^{n l}\left(\tilde{\delta}_{i}\right)=f\left(\tilde{\delta}_{i}\right) \tilde{\delta}_{i}$, which satisfies the constraints of nonlinear energy boundedness $0 \leq$ $f\left(\tilde{\delta}_{i}\right) \tilde{\delta}_{i} \leq \tilde{\delta}_{i}^{2}$ in the region of attraction $\left(\delta_{i}+\delta_{i}^{*}\right) \leq \pi$ as shown in Fig. 5. This feature, also discussed in [21] is important for convergence and stability criteria as it can be proved that the overall energy function of (2) has positive and strictly decaying behavior in this region, meaning the $x$ converges to the $x^{*}$.

\subsection{8-Bus Test Power System}

The effectiveness of the proposed hybrid controller is illustrated on a 5-machine 8-bus power system of Fig. 6. For this, dynamic equivalent frequency dependent models of the generators is developed using electromagnetic transient simulation (EMTP) software $P S C A D^{T M}$ [25]. This network consists of five equivalent generating units of which three generators G1, G2 ,and G4 are of larger size than G3 and G5. It has been identified that three of the five generators, G2, G3, and G5 are coherently grouped in one area, while generators G1 and G4 form a second area. Generator under our study, G3, is connected to a constant voltage bus through two parallel transmission lines. All the generators are equipped with governor, exciter, AVR, and PSS. G3 is also controlled by the proposed control structure. Parameters of all models and operating conditions are given in [25]. The generator, itself, is modeled as a fifth-order dynamic model.

\subsection{Proposed Control System Architecture}

The states chosen for monitoring the rotor angle stability to provide damping and synchronizing forces for oscillations as mentioned before are rotor angle and speed of G3 $\left(\delta_{3}, \dot{\delta}_{3}\right)$ and the control action is excitation voltage reference $\left(V_{e 3}\right)$. The output of the controller is augmented to the excitation system, mitigating the kinetic energy of the synchronous generator through the field energy control (Fig. 6). The input to the adaptive controller is the reference command based on linear characteristic equation

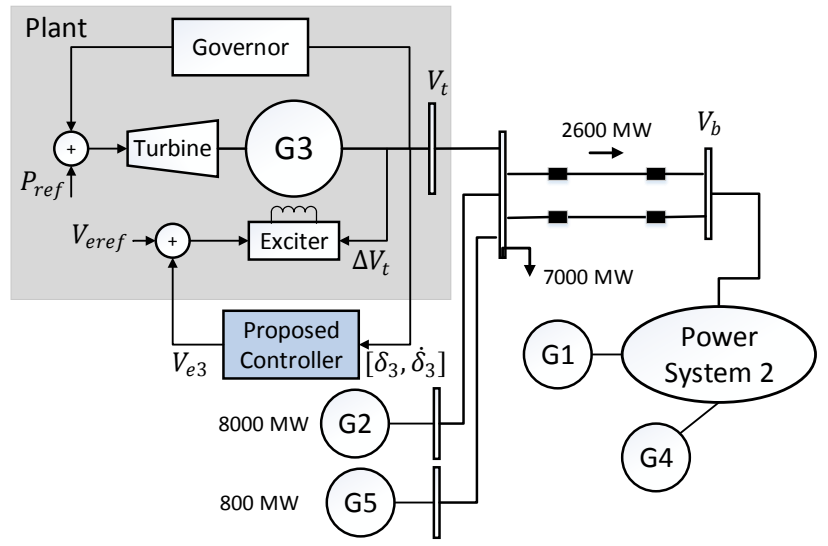

Figure 6: Structure of the 8-bus test power system

of the system, the reference model and the plant output. The reference model ensures that the generator is stable at all times and incorporates the dynamic preferences such as rise time and damping. Since the degree of the system is $n=2$ the error polynomial can be represented as $s^{2}+z_{1} s+z_{0}=0$. Setting $z_{0}=3.56$ and $z_{1}=2.67$, the roots can be placed at left-half plane in the frequency domain. The reference model was chosen to have a settling time of 3 seconds and a damping ratio of 0.707 , which is an industry accepted standard. Choosing $Q_{0}=\operatorname{diag}(1,1)$, and then by solving (4) we get $P=[0.6149,-0.5000 ;-0.5000,0.8539]$. Control coefficient is considered as $R=0.1$ and nonlinear coefficient $Q=[1 ; 0]$.

Specification of the ACD is as follows. The Action NN architecture consists of a three layer FFNN with two inputs, and a single hidden layer with 15 neurons and one output. The inputs are the generator angle and speed. These inputs are time delayed by $20 \mathrm{~ms}$ and, together with two previously delayed values, comprise the three inputs. The number of neurons in the hidden layer is identified in a heuristic manner. Critic NN has the specification with the output of one cost function. As discussed in previous sections, Identifier NN is used to learn the plant linear and nonlinear dynamics. The input to this NN is the states and the hybrid control. These inputs are time delayed by 20 ms and together with two previously delayed values, are used for learning.

For NN offline training, first, a set of random control inputs and respective outputs of the power system model for $100 \mathrm{sec}$ (5000 time steps) is fed into the identifier, and the outputs (the next time step's states) are extracted. The offline training as a batch learning algorithm is stopped when the Root Mean Square Error (RMSE) between the actual plant states and the identified ones, represented as $e^{I}$, reaches a user defined small number $(\epsilon=0.01)$. The learning rate of the NNs is $\alpha=0.01$. During online training, incremental learning is performed, in which, the identifier weights are adjusted based on the plant output and the reference model at each iteration. The same methodology is used for training the Action NN and Critic NN with their respective targets. Action training is started with the target output of MRAC. Furthermore, it is trained 

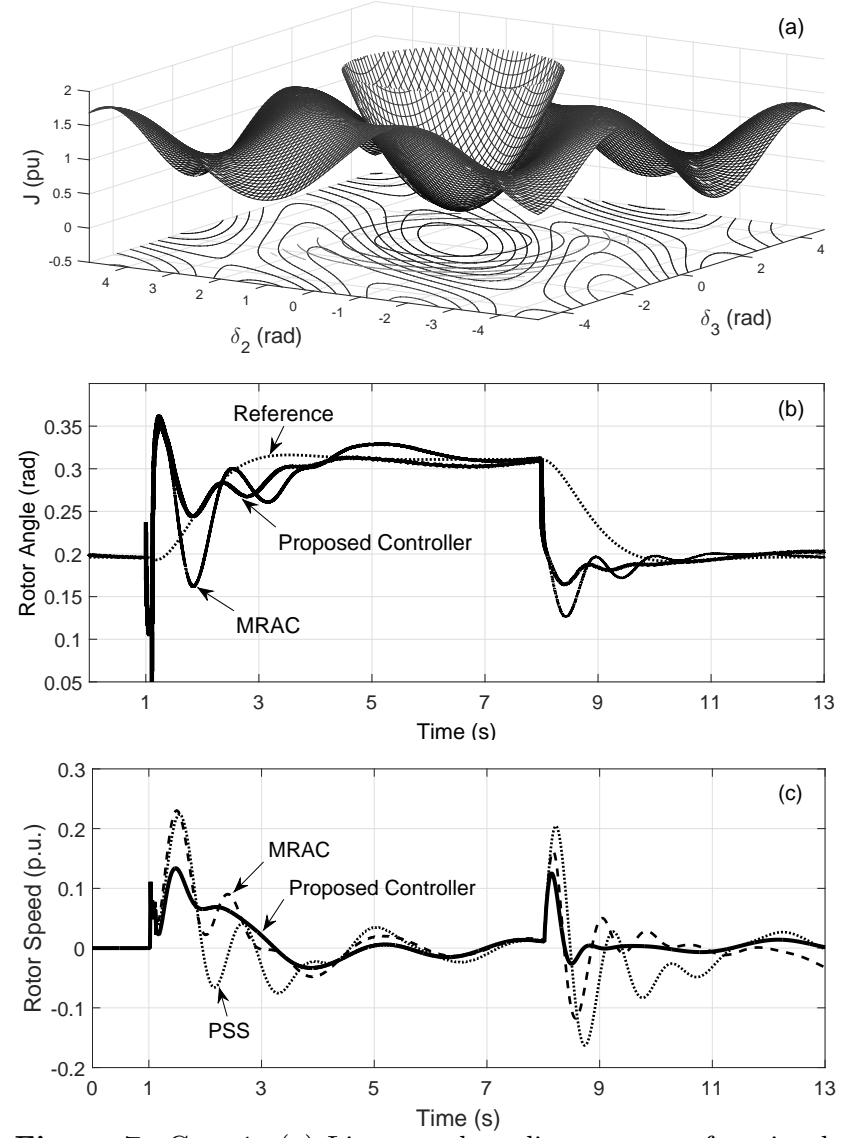

Figure 7: Case 1: (a) Linear and nonlinear energy function landscape as a function of $\delta_{2}$ and $\delta_{3}$, (b) G3 rotor angle deviation, $\delta_{3}$, (c) G3 rotor speed deviation, $\dot{\delta}_{3}$, with different controllers.

alongside the Critic NN, to perform optimization of the cost function. Utility function is developed based on $Q_{0}$, $R$, and $Q$ to monitor the energy function deviation and the power system stabilizers is updated to mitigate the oscillations. Once the critic weights have converged, the Action NN is connected to the generator's exciter to augment the MRAC. These controller's outputs are then fed to the plant through value priority NN block.

\subsection{Simulation Results}

\subsubsection{Case 1: Short Circuit}

In this case study, a $100 \mathrm{~ms}$ three-phase short circuit at the middle of one of the transmission lines between area 1 and area 2 is simulated. In order to assess the performance of the controllers, the transmission line is disconnected by means of breakers at each ends of the line. This line is then re-closed after $7 \mathrm{~s}$. This case study as a transient test effects all oscillatory modes of the system and the system topology. During this time, the system moves away from the pre-fault operating points $\delta_{2}^{0}=0.257$ and $\delta_{3}^{0}=0.1962$ and experiences a nonlinear post-fault transition dynamics after the re-closer action. This action causes a mismatch between generator reference and electrical powers, which leads to frequency increment and change in the tieline reactance. This yields to new operating points of $\delta_{2}^{*}=0.3683$ and $\delta_{3}^{*}=0.3111$. From Fig. 7a it can be observed that the initial and final operating points satisfy the nonlinear Lyapunov function constraints, and the energy is strictly decreasing in the region and converges to the post-fault optimal points. The reference value is obtained by means of the reference model response defined in previous section. Fig. $7 \mathrm{~b}$ shows the G3 rotor angle response of the system with the MRAC and the proposed adaptive learning-based controller. The NN-based RL controller is able to track the nonlinear dynamics and energy function, and enhance the performance of the controllers with respect to damping capability of $\dot{\delta}_{3}$ oscillation, as shown in Fig. 7c. As it can be seen from the figure, a notable damping improvement is gained, when the proposed controller with trained RL is installed on G3.

\subsubsection{Case 2: Voltage Reference Change}

In this test, a 0.05 p.u. step increase in exciter reference voltage of G3 is applied at $t=1 \mathrm{~s}$ and removed after $7 \mathrm{~s}$. The main purpose of this study is to show the capability of the value priority network in recognition of identification and performance criteria. In this case study, NNs with initial random weights is utilized for the RL-based controller. The terminal voltage response of G3 in the case of different controllers is shown in Fig. 8a. It can be seen that the proposed control scheme provides a quick, well-damped response in voltage. It can be noted that, the gain scheduler made a gradual shift from full supervision to full autonomy as the NNs acquired enough knowledge to reach the optimal performance target. Fig. 8b shows the corresponding proposed value priority functions for adaptive and RL controllers $\left(J^{v p \mid a d}, J^{v p \mid r}\right.$ ) and RL value priority trajectory $\left(\vartheta^{r}\right)$. As it can be noted, initially $J^{v p \mid r}$ is larger than $J^{v p \mid a d}$ due to identification error. However after learning it will lean towards RL controller. It can also be seen that this method allows the RL to reach the optimal weights faster with the help of supervisor, leading to lower RMSE of Action NN weights (Fig. 8c). The optimal weights are calculated based on 100 epochs of batch learning for this specific scenario.

\section{Implementation as a Wide-Area Controller}

Wide-Area Control (WAC) has gained momemtum due to the fact that inter-area response are more effectively damped using wide-area measurements and the technology advances in using Global Positioning System (GPS) and Phasor Measurement Unit (PMU) [26] allows us to develop such controllers. However, it is a known fact that modelbased Wide-Area Control (WAC) are not practical due to complexity in developing a wide-area scalable system model. Measurement based WAC on the other hand can use PMU signals but may incur certain time-delay and potential missing data information. This may eventually degrade the performance of such controllers and may even destabilize the system [7]. In this section, effective use of the proposed hybrid controller as a WAC is illustrated. 

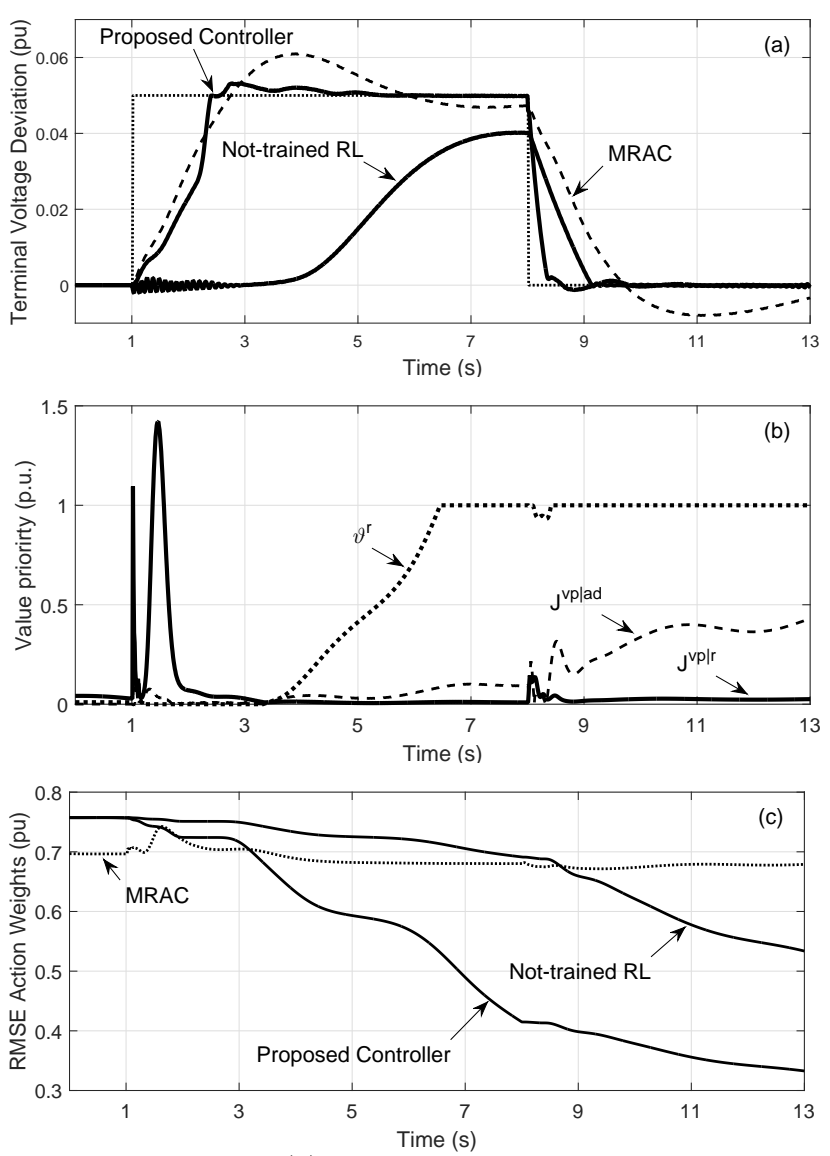

Figure 8: Case 2: (a) Dynamics of the G3 as terminal voltage deviation with different controllers, (b) value priority trajectory and elements, (c) RMSE of the control weights from the optimal weights.

Table 1: PSS Parameters for the IEEE 68-bus power system

\begin{tabular}{lcccccccc}
\hline \hline Generator & $K_{s}$ & $T_{s}$ & $T 1$ & $T_{2}$ & $T_{3}$ & $T_{4}$ & $T_{5}$ & $T_{6}$ \\
\hline$G_{1}: G_{8}, G_{10}: G_{12}$ & 20 & 15 & 0.15 & 0.04 & 0.15 & 0.04 & 0.15 & 0.04 \\
$G_{9}$ & 12 & 10 & 0.09 & 0.02 & 0.09 & 0.02 & 1 & 1 \\
\hline \hline
\end{tabular}

To illustrate the performance of the proposed approach as a WAC, tests are carrried out on 68-bus 16-machine IEEE test power system simulated in PST toolbox (see Fig. 9). A brief description of dynamic component modeling, and its governing equations are presented in [27]. Two types of excitation systems for the generators is considered: IEEE standard DC exciter (DC4B) and the standard static exciter (ST1A). In order to damp the local modes, excitation control of generators is supplemented with PSS with the parameters of Table 1 using the feedback signal of the local measurements of $\dot{\delta}_{i}$.

To reduce the size of the data set for training, initially generator coherent groups are developed (see Fig. 9). Further, commonly used center of angle/speed is found through the sensors as,

$$
\delta_{j}^{C}=\sum_{i} \delta_{i} M_{i} / \sum_{i} M_{i}, \quad \dot{\delta}_{j}^{C}=\sum_{i} \dot{\delta}_{i} M_{i} / \sum_{i} M_{i}
$$

where, $j$ is representative of the areas which is five in this case study and $i$ is the index of generator in that area. In

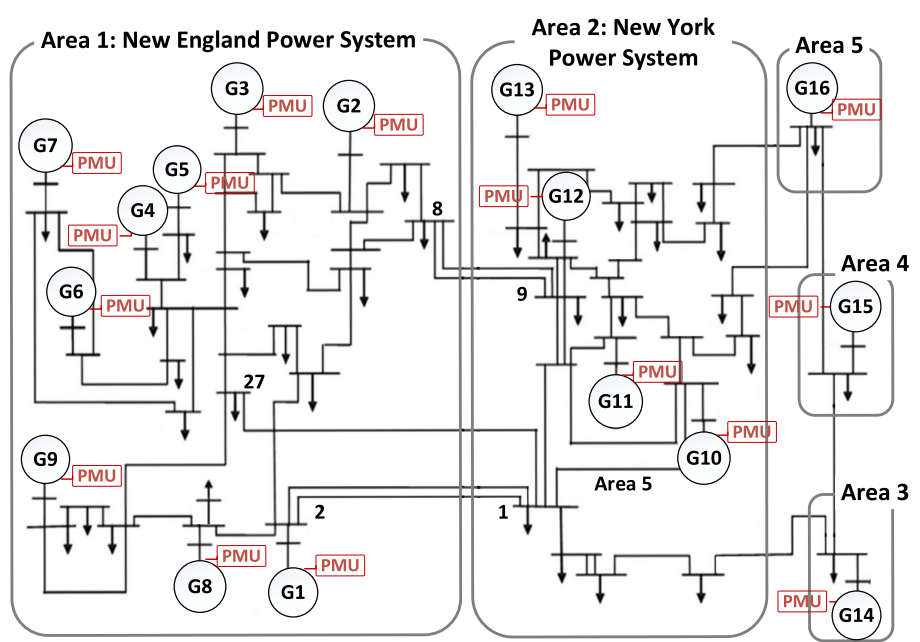

Figure 9: Single line diagram of the 68-bus 16-machine New England/New York power system.

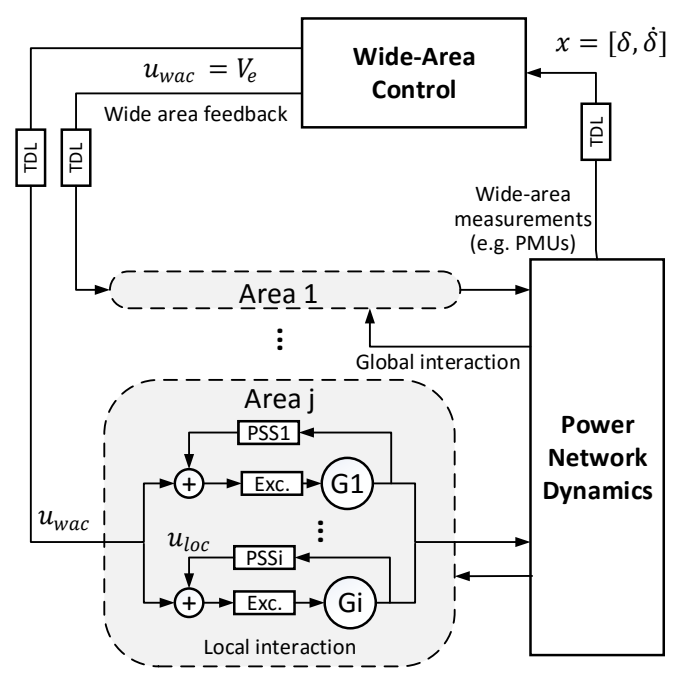

Figure 10: Wide-area control implementation design. (TDL: Time Delay Line)

this paper, it is assumed that each generator has a sensor that transmits signals to the WAC based on the defined objective functions, and WAC would send appropriate control signals to the generators excitation system. Fig. 10 illustrates the WAC implemented on the test power system. The wide-area NN Identifier, action and critic NNs are trained on the aggregated area signals, and control signals are calculated according to energy of each area. Parameters of the proposed WAC NNs is provided in Table 2. Communication system in this model can transmit timetagged phasor measurements $\left(\delta_{i}, \dot{\delta}_{i}\right)$ to WAC at the speed of $100 \mathrm{frames} / \mathrm{s}$; therefore, sampling time step is considered as $10 \mathrm{~ms}$ [26].

The main goal of this study is to evaluate the effectiveness of the proposed WAC scheme for damping the inter-area oscillations. For this, three tie-lines connecting the New England and New York power systems are disconnected sequentially. First, line 8-9 is disconnected allowing the RL to gain enough knowledge about the system dynamics and optimal policy. Next, line 1-27 is disconnected 
Table 2: Configuration of Neural Networks for the WAC

\begin{tabular}{lllc}
\hline \hline NN & $\begin{array}{l}\text { Inputs (Num- } \\
\text { ber of nodes) }\end{array}$ & $\begin{array}{l}\text { Outputs (Num- } \\
\text { ber of nodes) }\end{array}$ & $\begin{array}{l}\text { (Number of } \\
\text { hidden nodes) }\end{array}$ \\
\hline Identifier & $\delta^{C}, \dot{\delta}^{C}, V_{e}(45)$ & $\delta^{C}, \dot{\delta}^{C}(10)$ & $(60)$ \\
Action & $\delta^{C}, \dot{\delta}^{C}(30)$ & $V_{e}(5)$ & $(40)$ \\
Critic & $\delta^{C}, \dot{\delta}^{C}(30)$ & $J(5)$ & $(40)$ \\
\hline \hline
\end{tabular}
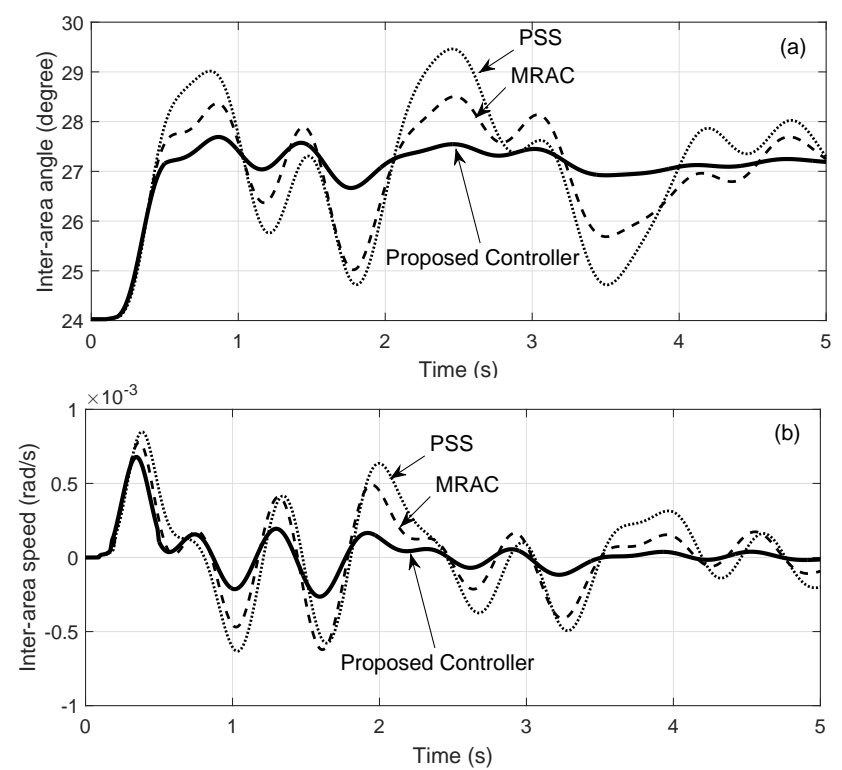

Figure 11: Dynamics of the IEEE 68-bus system in event 2, short circuit in tie-line 1-27, as inter-area 12 angle and speed oscillations.

due to a self-clearing $100 \mathrm{~ms}$ three-phase short circuit fault, which leads to severe mismatch between the energies of these two systems and angle deviation of $3.12^{\circ}$. Proposed controller and MRAC are designed based on global loop, which involves a multiple differential frequency signals of the selected areas with the uncertainty of the transmission delay. In this case study, it is assumed that the wide-area system consists of $100 \mathrm{~ms}$ transport delay. In order to predict the plant state vector at time step $k+10$, the values of state at time steps $k, k-10$, and $k-20$ are used. Fig. 11. depicts the time-domain performance of the controllers with $100 \mathrm{~ms}$ delayed data set. The local PSS acts on the local modes of oscillation with the frequency of $0.9824 \mathrm{~Hz}$, while the proposed WAC tracks the inter-area oscillation with the frequency of $0.6492 \mathrm{~Hz}$. Further, Fig. 12 depicts the Action and Critic NN performance in tracking their respective linear references and learning evoluation that minimize the actual Lyapunov function.

Finally, line 1-2 has been disconnected, leading to complete separation of these two areas. An eigenvalue analysis of the open-loop and closed-loop system with different control schemes was carried out for the events to examine the performance of the designed controller in terms of improving the damping ratios of the corresponding interarea mode. Parameters of the oscillations are derived by means of Prony analysis, which is listed in Table 3. It can be seen that the damping ratios of oscillations following the sequence of tie-lines outages has improved consider-
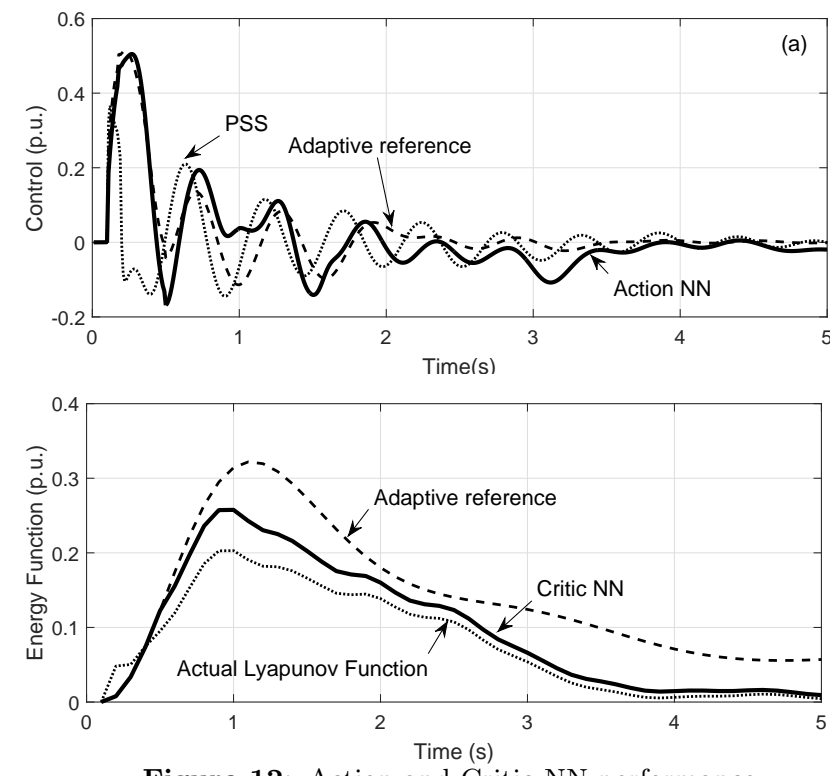

Figure 12: Action and Critic NN performance.

ably in presence of proposed hybrid controller. Additionally, Transient Energy function Index (TEI) (scaled based on "No control" case) has been listed for the case studies. This index, which is defined as the discounted cumulative value of utility function (24) over the simulation time period based on coherent signals of (34), reveals that transient energy has optimally been minimized with the proposed WAC.

\section{Conclusion}

A hybrid adaptive learning-based control design using Lyapunov stability function is presented in this paper for power system stabilization. The proposed architecture is feasible and show better damping and transient response than the linear adaptive controller acting alone. It is noted that the supervision of the linear controller can increase the speed of learning of reinforcement learning-based controller. The main advantage of this algorithm is that it is precise, feasible, and more reliable than other nonlinear adaptive controllers. Simulation results are presented to show the effectiveness of the proposed architecture in augmenting the MRAC installed on one of the generators on five-machine eight-bus two-area system to damp the oscillations. Furthermore, the proposed architecture is successfully implemented as WAC for inter-area oscillation and adaptation to new operating points.

\section{Appendix A. Proof of Theorem 1}

The convergence proof is based on ADP convergence proof in [24]. As critic training is based on the combination of errors of self-exploration and supervised action as defined in (28), we prove that the update method will always converge to the optimal value. Considering that $J_{i}^{r}\left(x_{k}\right)$ alone is a non-decreasing sequence for HDP [24], $\forall i: J_{i+1}^{r}\left(x_{k}\right) \geq J_{i}^{r}\left(x_{k}\right)$. Also it is proved that if $u_{k}^{a d}$ is a stabilizing and admissible control policy, then 
Table 3: Control performance in the cases of sequence of tie-line outages in terms of transient energy index (TEI) and damping factor of oscillations $(\zeta)$.

\begin{tabular}{c|cc|cc|cc|cr}
\hline \hline \multirow{2}{*}{ Outage of tie-line } & \multicolumn{2}{|c|}{ No control } & \multicolumn{2}{|c|}{ PSS } & \multicolumn{2}{c|}{ Adaptive WAC } & \multicolumn{2}{c}{ Proposed WAC } \\
\cline { 2 - 10 } & $T E I(\mathrm{pu})$ & $\zeta(\%)$ & \multicolumn{2}{|c|}{$T E I(\mathrm{pu})$} & $\zeta(\%)$ & $T E I(\mathrm{pu})$ & $\zeta(\%)$ & \multicolumn{2}{c}{$T E I(\mathrm{pu})$} & $\zeta(\%)$ \\
\hline Event 1 $(8-9)$ & 1 & 0.032 & 0.796 & 0.132 & 0.676 & 0.134 & $\mathbf{0 . 3 1 2}$ & $\mathbf{0 . 1 5 2}$ \\
\hline Event 2 $(1-27)$ & 1 & 0.017 & 0.789 & 0.034 & 0.601 & 0.045 & $\mathbf{0 . 2 8 0}$ & $\mathbf{0 . 0 7 5}$ \\
\hline Event 3 $(1-2)$ & 1 & 0.016 & 0.890 & 0.032 & 0.655 & 0.039 & $\mathbf{0 . 2 5 1}$ & $\mathbf{0 . 0 8 4}$ \\
\hline \hline
\end{tabular}

$\forall i: J_{i}^{r}\left(x_{k}\right) \leq J_{i}^{a d}\left(x_{k}\right) \leq J_{\max }\left(x_{k}\right)$. Thus the nonlinear energy function is bounded by the linear function (see Fig. 5) and the boundary conditions of $\vartheta^{r}=0$ and $\vartheta^{r}=1$ are stable. Hence the problem entitles the stability of the controller when $0<\vartheta^{r}<1$. We investigate three cases:

1. If $J_{i-1}^{r}\left(x_{k}\right)<J^{a d}\left(x_{k}\right)$, then the target update is in the same direction as the incremental update. This makes the output to converge to optimal value faster, and $\vartheta^{r}$ remains zero.

2. If $J_{i-1}^{r}\left(x_{k}\right) \geq J^{a d}\left(x_{k}\right)$, then $\vartheta^{r}$ is increased, and since $J_{i+1}^{r} \geq J_{i}^{r}$ is non-decreasing then for the following time sequence $J_{i}^{r} \geq J^{a d}$ holds.

3. If $J_{i-1}^{r}\left(x_{k}\right)>J^{a d}\left(x_{k}\right)$ and $\left|e_{C}\right|>\mid\left(J^{a d}-J_{i}^{r}\right)$, then the RL policy will get closer to the optimal value after few iterations of delay till $\vartheta^{r}=1$ and then completely latches to the RL controller.

The same method implies action convergence since they are directly connected to each other.

\section{References}

[1] P. Kundur, et. al, Definition and classification of power system stability ieee/cigre joint task force on stability terms and definitions 19 (3) (2004) 1387-1401.

[2] P. Kundur, M. Klein, G. Rogers, M. Zywno, Application of power system stabilizers for enhancement of overall system stability, Power Systems, IEEE Trans. on 4 (2) (1989) 614-626.

[3] A. Ghosh, G. Ledwich, O. Malik, G. Hope, Power system stabilizer based on adaptive control techniques, Power Apparatus and Systems, IEEE Transactions on (8) (1984) 1983-1989.

[4] Y. Wang, D. Hill, R. Middleton, L. Gao, Transient stability enhancement and voltage regulation of power systems, Power Systems, IEEE Transactions on 8 (2) (1993) 620-627.

[5] M. Mahmoudi, J. Dong, K. Tomsovic, S. Djouadi, Application of distributed control to mitigate disturbance propagations in large power networks, in: North American Power Symposium (NAPS), 2015, IEEE, 2015, pp. 1-6.

[6] A. P. W. W. D. Si, J.; Barto, Handbook of Learning and Approximate Dynamic Programming, Wiley-IEEE Press, 2004.

[7] S. Mohagheghi, G. Venayagamoorthy, R. Harley, Optimal wide area controller and state predictor for a power system, Power Systems, IEEE Transactions on 22 (2) (2007) 693-705.

[8] R. Yousefian, S. Kamalasadan, Design and real-time implementation of optimal power system wide-area system-centric controller based on temporal difference learning, Industry Applications, IEEE Transactions on PP (99) (2015) 1-1.

[9] G. K. Venayagamoorthy, R. G. Harley, D. C. Wunsch, Implementation of adaptive critic-based neurocontrollers for turbogenerators in a multimachine power system, Neural Networks, IEEE Transactions on 14 (5) (2003) 1047-64.

[10] M. Tofighi, M. Alizadeh, S. Ganjefar, M. Alizadeh, Direct adaptive power system stabilizer design using fuzzy wavelet neural network with self-recurrent consequent part, Applied Soft Computing 28 (2015) 514-526.
[11] K. Balasubramaniam, B. Luitel, G. Venayagamoorthy, A scalable wide area monitoring system using cellular neural networks, in: Proc. Int. Joint Conf. on Neural Networks (IJCNN), 2012, pp. $1-8$.

[12] S. Kamalasadan, G. Swann, R. Yousefian, A novel systemcentric intelligent adaptive control architecture for power system stabilizer based on adaptive neural networks, Systems Journal, IEEE 8 (4) (2014) 1074-1085.

[13] M. Bahita, K. Belarbi, Neural stable adaptive control for a class of nonlinear systems without use of a supervisory term in the control law, Journal of Engineering Science and Technology 7 (1) (2012) 97-118.

[14] G. Yen, P. DeLima, Improving the performance of globalized dual heuristic programming for fault tolerant control through an online learning supervisor, Automation Science and Engineering, IEEE Transactions on 2 (2) (2005) 121-131.

[15] R. Sutton, A. Barto, Reinforcement Learning: An Introduction 9 (5) (1998) 1054-1054.

[16] P. J. Werbos, Advanced forecasting methods for global crisis warning and models of intelligence, General Systems Yearbook 22 (1977) 25-38.

[17] R. Yousefian, S. Kamalasadan, Value priority based optimal power system stabilization of generating resources using local and global controllers, in: Power Energy Society General Meeting, 2015 IEEE, 2015, pp. 1-5.

[18] M. T. Rosenstein, A. G. Barto, Supervised learning combined with an actor-critic architecture, Dept. of Comput. Sci., Univ. of Massachusetts, Tech. Rep (2002) 02-41.

[19] R. A. Jacobs, M. I. Jordan, S. J. Nowlan, G. E. Hinton, Adaptive mixtures of local experts, Neural computation 3 (1) (1991) 79-87.

[20] N. Kakimoto, Y. Ohsawa, M. Hayashi, Transient stability analysis of multimachine power system with field flux decays via lyapunov's direct method, Power Apparatus and Systems, IEEE Transactions on (5) (1980) 1819-1827.

[21] T. Vu, K. Turitsyn, Lyapunov functions family approach to transient stability assessment, Power Systems, IEEE Transactions on PP (99) (2015) 1-9.

[22] M. A. Duarte, K. S. Narendra, Combined direct and indirect approach to adaptive control, Automatic Control, IEEE Transactions on 34 (10) (1989) 1071-1075.

[23] S. Fabri, V. Kadirkamanathan, Functional Adaptive Control: An Intelligent Systems Approach, Advanced Manufacturing Series.

[24] A. Al-Tamimi, F. Lewis, M. Abu-Khalaf, Discrete-time nonlinear hjb solution using approximate dynamic programming: Convergence proof 38 (4) (2008) 943-949.

[25] D. Chaturvedi, O. Malik, Experimental studies of a generalized neuron based adaptive power system stabilizer, Soft Comput. 11 (2) (2007) 149-155.

[26] C. W. Taylor, D. C. Erickson, K. E. Martin, R. E. Wilson, V. Venkatasubramanian, Wacs-wide-area stability and voltage control system: R\&d and online demonstration, Proceedings of the IEEE 93 (5) (2005) 892-906.

[27] IEEE 68 bus system benchmark model. URL http://www.sel.eesc.usp.br/ieee/ 